\title{
Die Logistik im Fokus empirischer Analysen: Arbeitsbedingungen und Arbeitsfähigkeit in der Lagerwirtschaft sowie den Post- und Zustelldiensten
}

\author{
Mathias Certa ${ }^{1} \cdot$ Tim Schröder ${ }^{2}$ \\ Online publiziert: 6. November 2020 \\ (c) Der/die Autor(en) 2020
}

\section{Zusammenfassung}

Die zunehmende Digitalisierung führt zu veränderten Arbeitsbedingungen in verschiedenen Bereichen der Logistik, wie der Intralogistik und den Post- und Zustelldiensten. Für beide Berufsgruppen gelten die Arbeitsbedingungen als äußerst belastend. Allerdings sind die Zusammenhänge mit der Arbeitsfähigkeit bislang nicht hinreichend untersucht. Ziel ist es deshalb, diese Zusammenhänge anhand empirischer Analysen und auf theoretischer Basis eines erweiterten AnforderungsRessourcen-Modells zu erklären und somit Ansatzpunkte für eine menschengerechte Arbeitsgestaltung aufzuzeigen. Datengrundlage ist die von der Bundesanstalt für Arbeitsschutz und Arbeitsmedizin durchgeführte Arbeitszeitbefragung. Anhand von Gruppenvergleichen und Regressionsmodellen werden beide Berufsgruppen miteinander verglichen. Ansätze für eine menschengerechte Gestaltung von Arbeitssystemen können für beide Berufsgruppen vor allem bei organisatorischen Arbeitsbedingungen wie der sozialen Unterstützung oder eines auf Anerkennung und Rollenklarheit basierenden Betriebsklimas ausgemacht werden.

Praktische Relevanz Die vorliegenden Analysen können als Grundlage für eine menschengerechte Gestaltung von Arbeitssystemen je nach den spezifischen Bedingungen in der jeweiligen Berufsgruppe dienen. Sie verdeutlichen besonders das Bedingungsverhältnis von technischen und sozialen Aspekten und zeigen darüber hinaus für beide untersuchten Berufsgruppen sowohl bei der technischen Gestaltung der Arbeitssysteme als auch mit Blick auf organisationale Ressourcen Ansatzpunkte für Interventionen auf.

Schlüsselwörter Logistik · Lagerwirtschaft · Post- und Zustelldienste · Arbeitsfähigkeit · Arbeitsbedingungen

\section{Focus on logistics: working conditions and work ability in warehousing as well as postal and delivery services}

\section{Abstract}

Digitalisation leads to changing working conditions in various areas of logistics, including intralogistics as well as postal and delivery services. For both groups, working conditions are considered extremely stressful. However, the interplay between these and work ability have not yet been sufficiently investigated. To explain this relationship, an extension of the job demands-resources model as theoretical foundation as well as data from Working Time Survey conducted by the Federal Institute for Occupational Safety and Health for empirical testing is used. Both intralogistics and postal and delivery services are compared with each other by group comparisons and regression models.

Mathias Certa

certa.mathias@baua.bund.de

1 Bundesanstalt für Arbeitsschutz und Arbeitsmedizin, Friedrich-Henkel-Weg 1-25, 44149 Dortmund, Deutschland
2 Forschungsinstitut gesellschaftlicher Zusammenhalt (FGZ), Universität Bremen, Mary-Somerville-Str. 9, 28359 Bremen, Deutschland 
Practical Relevance The findings presented in this article can serve as a basis for a preventive design of work systems. In particular, they illustrate the conditional relationship between technical and social aspects. In addition, they show specific starting points for interventions for both occupational groups regarding the technical design of work systems as well as organisational resources.

Keywords Logistics $\cdot$ Warehousing $\cdot$ Postal and delivery service $\cdot$ Work ability $\cdot$ Working conditions

\section{Einleitung}

Die zunehmende Digitalisierung führt zu veränderten Arbeitsbedingungen in verschiedenen Bereichen der Logistik. So zeigt sich der technologische Fortschritt zum Beispiel durch den zunehmenden Einsatz von miteinander verknüpften Sensor- und Kommunikationstechnologien, von technischen Assistenzsystemen oder von Robotern (Ortmann und Walker 2019). Im Bereich des Kurier-, Express und Paketmarktes erfährt zudem insbesondere das Sendungsvolumen innerhalb Deutschlands im Zuge der Digitalisierung und der damit einhergehenden veränderten Kaufmentalität ein stetiges Wachstum und hat sich innerhalb der letzten zwei Jahrzehnte verdoppelt (Bundesverband Paket und Expresslogistik 2019). All diese Veränderungen führen zu einer Transformation des Arbeitsalltages der Beschäftigten in der Logistik (Straub et al. 2017). Die Bereiche der Post- und Zustelldienste - im Nachfolgenden kurz Postberufe - sowie der Lagerwirtschaft bzw. der Intralogistik haben darüber hinaus die Gemeinsamkeit, dass sie im Wesentlichen aus Einfacharbeit in Form des Kommissionierens, des Sortierens und Transportierens von Waren bestehen und - insbesondere die Lagerwirtschaft - zentrale Tätigkeitsbereiche für Einfachbeschäftigte darstellen (Ortmann und Walker 2019). Während in der Intralogistik der unternehmensinterne Warenfluss im Vordergrund steht, kommt in den Postberufen zu einem Gutteil die Zustellung an Kunden hinzu.

Die Arbeitsbedingungen in beiden Berufsgruppen gelten als äußerst belastend (Hartwig und Mamrot 2018; Zanker 2018). In erster Linie handelt es sich um körperlich schwere Arbeit unter ungünstigen Umfeldbedingungen wie Lärm, Nässe oder Kälte. Auch die psychisch-informatorische Belastung durch Leistungsdruck, direkte Kontrolle durch Vorgesetzte sowie Schicht- und Wochenendarbeit ist hoch. Langfristig können sich hieraus negative Auswirkungen auf die Arbeitsfähigkeit ergeben. So gehören die Lagerwirtschaft und Postberufe zu den Berufsgruppen mit den häufigsten Fehltagen aufgrund von Arbeitsunfähigkeit (Knieps und Pfaff 2017). Ursache sind oft Muskel-SkelettErkrankungen (v. a. Rückenschmerzen) sowie Arbeitsunfälle und Verletzungen, häufig aber auch psychische Störungen (v. a. Depressionen). Dies wiederum wirkt auf die Unternehmen zurück und verringert deren Wettbewerbsfähigkeit.

Die Frage, welche konkreten Arbeitsbedingungen mit einer verringerten Arbeitsfähigkeit einhergehen und welche sie - mit Blick auf menschengerechte Arbeitsgestaltung auch fördern können, ist für beide Berufsgruppen bislang jedoch nicht hinreichend untersucht. So lassen sich die Arbeitsbedingungen in negativ bewertete Arbeitsanforderungen (Stressoren), aber auch positiv bewertete Anforderungen (Herausforderungen) sowie organisationale und individuelle Ressourcen differenzieren. Die vorliegende Untersuchung dient der empirischen Überprüfung dieser $\mathrm{Zu}$ sammenhänge, um darauf aufbauend Ansatzpunkte für eine menschengerechte Arbeitsgestaltung aufzeigen zu können. Datengrundlage ist die deutschlandweit durchgeführte Arbeitszeitbefragung der Bundesanstalt für Arbeitsschutz und Arbeitsmedizin (BAuA). Es wird damit an die deskriptiven Analysen von Hartwig und Mamrot (2018) zur Intralogistik angeknüpft und diese in dreifacher Hinsicht erweitert. Erstens hilft eine theoretische Fundierung durch das Anforderungs-Ressourcen-Modell dabei, Arbeitsbedingungen gezielt danach zu differenzieren, ob sie zur Gefährdung oder Förderung der Arbeitsfähigkeit beitragen. Zudem können über den Vergleich mit den empirisch gut bestätigten Modellannahmen, die empirischen Ergebnisse entweder generalisiert oder berufsgruppenspezifische Besonderheiten identifiziert werden. Multiple Regressionen erlauben dabei zweitens, den Zusammenhang von Arbeitsbedingungen und Arbeitsfähigkeit durch Kontrolle jeweiliger Drittvariablen genauer zu erfassen. Drittens ermöglicht der Vergleich zwischen Lagerwirtschafts- und Postberufen aufgrund deren ähnlich gelagerter Tätigkeiten und Arbeitsgestaltung Besonderheiten in den Arbeitsbedingungen abzugrenzen und spezifische Unterschiede in den Auswirkungen klar zu umreißen.

\section{Theoretische Grundlage}

Als Erklärungsgrundlage dient das in Theorie und Praxis des Arbeits- und Gesundheitsschutzes international weit verbreitete Anforderungs-Ressourcen-Modell (,,job demands-resources model") (Demerouti et al. 2001; Bakker und Demerouti 2017; Schaufeli und Taris 2014). Dabei kommt die erweiterte Form zur Anwendung, welche die Anforderungen nach Stressoren (,hindrances") und Herausforderungen (,challenges") differenziert (Van Den Broeck et al. 2010; Lepine et al. 2005; Crawford et al. 2010). Das Modell dient als ein flexibles, heuristisches und deskriptives 
Rahmenmodell mit klar abgegrenzten Begriffsdefinitionen, das verschiedene andere Modelle integriert (Schaufeli und Taris 2014; Melzer und Hubrich 2014, S. 10). Es findet zudem berufs- und branchenübergreifend auf vielen Feldern Anwendung, etwa in der Transportbranche (Semeijn et al. 2019) oder zur umfassenden Identifizierung der allgemeinen Schlüsselfaktoren psychischer Gesundheit (Rothe et al. 2017). Empirisch ist es weitgehend bestätigt (Lesener et al. 2019). Dabei beansprucht es sowohl allgemeingültig zu sein als auch spezifische Anforderungen und Ressourcen erfassen zu können. Schließlich ist es für die Frage menschengerechter Arbeitsgestaltung besonders relevant, da im Sinne der positiven Psychologie oder Salutogenese (Antonovsky 1988) auch positiv bewertete Bedingungen des Erhalts von Arbeitsfähigkeit und Gesundheit adressiert werden (Melzer und Hubrich 2014).

Die zentrale Begrifflichkeit des Modells bildet die Unterscheidung von Anforderungen und Ressourcen. Erstere gelten als physische, informatorische oder soziale Aspekte der Arbeitsstelle, die notwendig eine physische oder mentale Anstrengung erfordern und darum mit entsprechenden Kosten verbunden sind (Demerouti et al. 2001). Gemäß dem erweiterten Modell werden die Anforderungen in Anlehnung an die transaktionale Stresstheorie weiter differenziert. Als Stressoren gelten Arbeitsanforderungen dann, wenn sie als Hindernisse auf dem Weg zu Zielerreichung und Bedürfnisbefriedigung wahrgenommen werden und darum Arbeitsfähigkeit und Gesundheit gefährden. Zu Herausforderungen werden sie, wenn sie der Zielerreichung und Bedürfnisbefriedigung gerade zuträglich sind und deren Kosten deshalb in Kauf genommen werden. Sie können damit einerseits aufgrund der Verausgabung von Energie die Arbeitsfähigkeit und Gesundheit gefährden, dienen aber zugleich auch der Motivation und damit dem Wohlbefinden. So gilt die Arbeitsintensität (hohes Arbeitspensum, Zeitdruck) als Herausforderungen, insofern sie mit einem Gefühl der Beherrschung einhergeht. Erst dann, wenn sie mit kognitiver Irritation assoziiert sind, man etwa noch zuhause an die Arbeit denkt, werden sie zu Stressoren (Widmer et al. 2012). Als ebenso ambivalent gilt die Nutzung digitaler Technologien. Diese werden von Beschäftigten durchaus positiv bewertet (Härtwig und Sapronova 2020). Ein Grund dafür sind vermutlich die damit verbundenen kognitiven Anforderungen, wie die Verbesserung bestehender bzw. neue Aufgaben, die sich positiv auf Arbeitszufriedenheit und Gesundheit auswirken (Meyer und Hünefeld 2018). Störungen im Arbeitsfluss, bspw. durch E-Mail-Verkehr oder eine ständige Erreichbarkeit, gelten hingegen eher als Stressoren (Korunovska und Spiekermann 2019). Sie können u.a. zu digital bedingtem Stress führen (Gimpel et al. 2019).

Ähnlich wie die Herausforderungen sind auch die Ressourcen als Mittel zur Zielerreichung mit einer positiven Bewertung verknüpft. Der Unterschied besteht darin, dass sie nicht mit notwendigen Arbeitsprozessen einhergehen, sondern Möglichkeiten darstellen, die in Anspruch genommen werden können, aber nicht müssen. Den Ressourcen kommt daher ein weniger ambivalenter Charakter als den Herausforderungen zu.

Die Unterscheidung von Anforderungen in Form von Stressoren und Herausforderungen sowie Ressourcen erlaubt es, klare Thesen zu den physischen und psychischen Auswirkungen auf die Person $\mathrm{zu}$ formulieren. Üblicherweise wird von einem doppelten Prozess ausgegangen: die Stressoren führen einerseits in einem Prozess der Verausgabung von Energie zu negativem Wohlbefinden (,strain“) in Form von Erschöpfung und langfristig $\mathrm{zu}$ schlechter Gesundheit. Die Herausforderungen und Ressourcen führen andererseits zu einer erhöhten Motivation, zu Engagement und Bindung an den Betrieb. Zugleich besteht aber ein enger Zusammenhang beider Prozesse (Schaufeli und Taris 2014). So wird etwa angenommen, dass Ressourcen den Zusammenhang von Stressoren und schlechter Gesundheit abfedern können. Sowohl eine schlechte Gesundheit als auch eine geringe Arbeitszufriedenheit und Betriebsbindung verringern zudem die Arbeitsleistung. Im Fokus der folgenden Untersuchung steht deshalb nur eine abhängige Variable: die Arbeitsfähigkeit (Ilmarinen et al. 2008). Es handelt sich um ein mehrdimensionales Konstrukt, das sowohl Aspekte der Gesundheit als auch der Motivation enthält und sich mit dem Anforderungs-Ressourcen-Modell gut fassen lässt. Arbeitsfähigkeit ist definiert als die Fähigkeit einer Person, ihre Tätigkeit unter Berücksichtigung der Arbeitsanforderungen und Ressourcen zu verrichten und somit das Ergebnis der Interaktion von Person und Arbeitsumwelt (Ebener und Hasselhorn 2016; McGonagle et al. 2014). Sie wird im Anforderungs-Ressourcen-Modell als positives Ergebnis der Ausbalancierung von Anforderungen und Ressourcen gewertet und ist damit für die Frage menschengerechter Arbeitsgestaltung besonders relevant (Schaufeli und Taris 2014).

Das zugrundeliegende Modell und seine Operationalisierung in konkrete manifeste Variablen und latente Faktoren ist in Abb. 1 dargestellt. Die Variablen und Faktoren werden im folgenden Kapitel zu Daten und Methoden genauer vorgestellt. Unterschieden werden hier zunächst Stressoren und Herausforderungen als zwei Formen von Anforderungen. Erstere beschreiben negativ bewertete Anforderungen der Tätigkeit oder des Arbeitsumfeldes. Hierunter werden nachfolgend die Aspekte physischer Tätigkeiten, des physischen und sozialen Arbeitsumfeldes, der Arbeitszeitlage sowie der Veränderung des Arbeitsumfeldes gefasst. Ambivalenter zu betrachten sind die Anforderung eines Terminund Leistungsdrucks, des schnellen Arbeitens, des Multitaskings sowie der Nutzung moderner Informations- und Kommunikationstechnologien. Während Stressoren sich eindeutig negativ auf die Arbeitsfähigkeit auswirken sollten, sind 


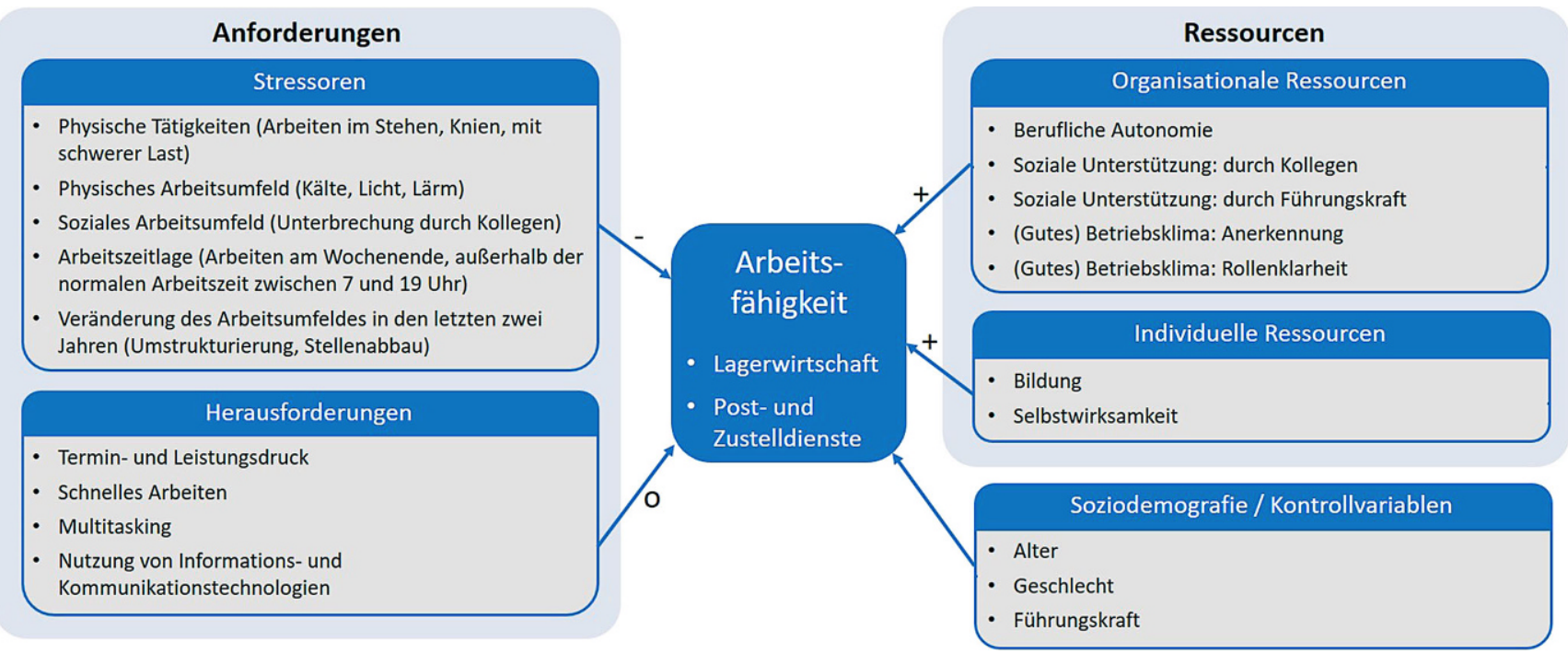

Abb. 1 Anforderungs-Ressourcen-Modell der Arbeitsfähigkeit

Fig. 1 Job demands-resources model of work ability

für die Herausforderungen zumindest keine eindeutigen Ergebnisse zu erwarten. Denkbar ist, dass sich der negativ bewertete Arbeitsaufwand und die positiv bewerteten Erträge in Form von Motivation neutralisieren und sich somit kein Effekt auf die Arbeitsfähigkeit zeigt. In welcher Form die hier beschriebenen ambivalenten Herausforderungen konkret Einfluss auf die Arbeitsfähigkeit in den beiden Berufsgruppen haben, kann einen wesentlichen Beitrag zur Entwicklung von menschengerechten Gestaltungsansätzen ausmachen. Von den Anforderungen werden organisationale Ressourcen (wie soziale Unterstützung) und individuelle Ressourcen (Selbstwirksamkeit) unterschieden. Beides sollte sich positiv auf die Arbeitsfähigkeit auswirken. Als individuelle sozio-demografische Kontrollvariablen wurden das Alter, das Geschlecht und das Ausüben einer Führungstätigkeit berücksichtigt.

Neben diesen sich aus dem Anforderungs-RessourcenModell ergebenden allgemeinen Annahmen werden im Folgenden keine spezifischen Hypothesen für die hier untersuchten Berufsgruppen formuliert. Hierzu mangelt es schlichtweg sowohl an theoretischen Erkenntnissen als auch an vergleichender empirischer Forschung. Im Fokus dieser Studie steht demnach eine explorative Vorgehensweise: ein Vergleich der Anforderungen und Ressourcen und der damit einhergehenden Arbeitsfähigkeit in den beiden Berufsgruppen mit den Modellannahmen. Erst im Nachgang lassen sich spezifische Aussagen zur menschengerechten Arbeitsgestaltung in den jeweiligen Berufsgruppen angesichts der besonderen Arbeitsbedingungen treffen.

\section{Daten und Methoden}

\subsection{Datengrundlage}

Als Grundlage für die Untersuchungen dient die als $\mathrm{Pa}$ nelstudie angelegte Arbeitszeitbefragung der BAuA. Diese zielt ab auf die repräsentative Erfassung und Beschreibung der körperlichen und mentalen Arbeitsbelastungen in $\mathrm{Zu}$ sammenhang mit der Gestaltung der Arbeitszeit sowie den Arbeitsbedingungen in Deutschland (Häring et al. 2016). Für die Auswertungen werden die ersten beiden Befragungswellen aus den Jahren 2015 und 2017 verwendet.

Für beide Wellen setzt sich die Grundgesamtheit der Erstbefragten (gesamte Stichprobe aus dem Jahr 2015, Auffrischerstichprobe aus dem Jahr 2017) aus Personen ab 15 Jahren zusammen, die in Privathaushalten leben und mindestens einer entgeltlichen Erwerbstätigkeit von mindestens $10 \mathrm{~h}$ pro Woche nachgehen. Auf dieser Grundlage erfolgten computerunterstützte Telefoninterviews (CATI), welche anhand zufällig generierter Festnetz- und Mobilfunknummern durchgeführt wurden. Für die erste Welle der Arbeitszeitbefragung im Jahr 2015 wurden 20.030 auswertbare Interviews generiert (Häring et al. 2016). Von diesen konnten in der zweiten Welle im Jahr 20177446 Personen erneut befragt werden. Allerdings waren zu diesem Zeitpunkt davon 799 Befragte nicht erwerbstätig, sodass für insgesamt 6647 Befragte Daten aus beiden Befragungszeitpunkten für die Analysen vorliegen. Diese wurden zudem aufgrund der hohen Panelmortalität ergänzt durch eine Auffrischerstichprobe von 3013 erstbefragten Personen. Somit liegen für die zweite Welle insgesamt 10.459 auswertbare Interviews vor (Häring et al. 2018). 


\subsection{Stichprobenselektion}

Im Fokus der Analysen stehen die Arbeitsbedingungen der Beschäftigten innerhalb der Lagerwirtschafts- sowie der Postberufe. Zur Selektion beider Berufsgruppen wurde die Klassifikation der Berufe 2010 herangezogen. Diese ist in fünf Gliederungsebenen unterteilt, von einer groben Differenzierung nach zehn unterschiedlichen Berufsbereichen bis hin zu 1286 Berufsgattungen als feinste Abstufung (Bundesagentur für Arbeit 2011). Anhand letzterer wurden die beiden zu untersuchenden Berufsgruppen identifiziert: die Gruppe der Lagerwirtschaft setzt sich zusammen aus Erwerbstätigen, welche als Helfer oder Fachkraft in der Lagerwirtschaft beschäftigt sind. Helfer oder Fachkräfte innerhalb der Post- und Zustelldienste wurden zur Subgruppe der Postberufe zusammengefasst. Weitere Selektionskriterien waren der Beschäftigungsstatus sowie das Alter. So wurden alle Personen aus den Analysen ausgeschlossen, die nicht abhängig beschäftigt sowie älter als 67 Jahre und somit im Rentenalter noch erwerbstätig waren. Für die Berufsgruppe der Beschäftigten in der Lagerwirtschaft ergab sich somit eine Fallzahl von 348 (Jahr 2015) bzw. 129 (Jahr 2017) Personen, während die Postberufe 121 (Jahr 2015) bzw. 52 (Jahr 2017) Personen umfasste (s. Tab. 1). Die Gruppe der restlichen Beschäftigten setzte sich für das Jahr 2015 aus 17.325 Personen und für das Jahr 2017 aus 8386 Personen zusammen. Diese Gruppen beinhalteten keine Beschäftigten aus den Lagerwirtschafts- oder Postberufen. Bei einem Vergleich zwischen der Lagerwirtschaft und den restlichen Beschäftigten beinhaltete letztere Gruppe somit nicht Personen, die in einem Postberuf arbeiten.

\subsection{Analysemethoden}

Aufgrund der geringen Fallzahlen - insbesondere in der Gruppe der Postberufe - war eine besonders sparsame Modellbildung erforderlich. So wurden im Vorfeld der Auswertung Faktorenanalysen durchgeführt, um Variablen zusammenfassen und Informationen bündeln zu können. Diese beruhten auf dem schiefwinkligen Oblimin-Rotationsverfahren, wodurch Abweichungen von der Orthogonalität zugelassen wurden. Für die im Nachfolgenden berichteten extrahierten Faktoren lagen die gängigen Maße für die Güte der Analysen wie Cronbachs Alpha (zwischen 0,63 und
$0,86)$ sowie dem Kaiser-Meyer-Olkin-Kriterium (zwischen 0,70 und 0,86 ) in vertretbaren Bereichen.

Zur Überprüfung auf signifikante Unterschiede zwischen den Gruppen der Lagerwirtschaft, der Postberufe sowie den restlichen Beschäftigten hinsichtlich deren Arbeitsbedingungen und Arbeitsfähigkeit wurden Gruppenvergleiche durchgeführt. Für einen aussagekräftigeren Vergleich der einzelnen Berufsgruppen mit der Gruppe der restlichen Beschäftigten wurde für letztere ein Subdatensatz mit $495 \mathrm{Be}-$ schäftigten gebildet, der hinsichtlich der Verteilung in den Variablen Alter, Bildungsniveau und Klassifikation der Berufe dem Gesamtdatensatz ähnelt. Da darüber hinaus den Daten bei ungleichen Gruppengrößen keine Normalverteilung zugrunde lag, wurde auf nicht-parametrische Testverfahren zurückgegriffen. Für den Vergleich von Variablen mit ordinalem Messniveau fand der Mann-Whitney-U-Test Anwendung. Dieser wurde sowohl für die Vergleiche zwischen den verschiedenen Berufsgruppen für das Jahr 2015 verwendet als auch für die Vergleiche innerhalb der zwei im Fokus stehenden Berufsgruppen zwischen den Jahren 2015 und 2017. Für letztere wurde auf Messverfahren für unabhängige Stichproben zurückgegriffen, da lediglich eine geringe Anzahl an Personen mit Informationen in beiden Zeiträumen vorlag (70 Personen in der Lagerwirtschaft und 27 Beschäftigte in den Postberufen). Darüber hinaus wurde zur zusätzlichen Einschätzung der Bedeutsamkeit der auftretenden Gruppenunterschiede die Effektstärke $\mathrm{r}$ berechnet (Pallant 2007). Nach Cohen (1988) geben Werte um 0,1 einen kleinen, um 0,3 einen mittleren und über 0,5 einen starken Effekt an. Dichotom ausgeprägte Variablen wurden anhand des $\mathrm{Chi}^{2}$-Tests auf signifikante Unterschiede hin überprüft und Cramers- $\mathrm{V}$ als Effektstärke herangezogen.

Zudem wurden abschließend verschiedene multiple lineare Regressionsmodelle gerechnet, um beim Zusammenhang von Anforderungen und Ressourcen mit der Arbeitsfähigkeit den Einfluss von beobachteten Drittvariablen kontrollieren zu können. So wurden für die beiden Gruppen separat Regressionen mit allen im Modell befindlichen Anforderungen und Ressourcen durchgeführt, um die spezifische Wirkung einzelner Faktoren innerhalb der Berufsgruppen erfassen zu können.
Tab. 1 Übersicht über die Fallzahlen nach Berufsgruppe und Erhebungsjahr Table 1 Number of cases by occupational group and survey year

\begin{tabular}{llllll}
\hline & 2015 & & 2017 & \\
\cline { 2 - 3 } & Ungewichtet & Gewichtet & Ungewichtet & Gewichtet \\
\hline Lagerwirtschaft & 348 & 521 & 129 & 223 \\
Postberufe & 121 & 141 & 52 & 55 \\
Vergleichsgruppe & 17.475 & 17.488 & 8386 & 8464 \\
Gesamt & 17.944 & 18.150 & 8567 & 8742 \\
\hline
\end{tabular}




\subsection{Operationalisierung des Erklärungsmodells}

Zentrale abhängige Variable in den Regressionsmodellen ist ein Item des „Work Ability Index“ (Ebener und Hasselhorn 2016): die subjektive Bewertung der eigenen Arbeitsfähigkeit. Sie wurde auf einer Skala von 0 (,arbeitsunfähig“) bis 10 (die „beste, je erreichte Arbeitsfähigkeit“) gemessen. Die Regressionskoeffizienten beziehen sich damit auf eine Veränderung der Arbeitsfähigkeit um einen Skalenwert.

\subsubsection{Anforderungen}

Stressoren Die physischen Anforderungen der Tätigkeiten (Cronbachs Alpha: 0,79) sowie des Arbeitsumfeldes (Cronbachs Alpha: 0,71) konnten in einer Faktorenanalyse klar als zwei Faktoren mit jeweils drei Items identifiziert werden. Somit gehen nicht die Einzelitems, sondern die vorhergesagten Faktorwerte in die Regressionsanalysen ein. Die physischen Tätigkeitsanforderungen beziehen sich auf im Stehen, in kniender/gebückter Körperhaltung durchgeführte oder mit dem Tragen schwerer Lasten verbundene Tätigkeiten. Stressoren des physischen Arbeitsumfeldes sind unter anderem Kälte, Hitze und Feuchtigkeit, ungünstige Lichtverhältnisse oder Lärm. Die jeweiligen Items wurden auf einer vierstufigen Likert-Skala (1=häufig; $2=$ manchmal; $3=$ selten; $4=$ nie) abgefragt und für die Analysen umgepolt, sodass ein hoher Wert einer hohen Belastung entspricht. Ein Stressor des sozialen Arbeitsumfeldes ist Störungen oder Unterbrechungen bei der Arbeit durch Kollegen. Dieser wurde auf einer vierstufigen Likert-Skala ( $1=$ nie; $2=$ selten; $3=$ manchmal; $4=$ häufig) gemessen.

Die ungünstige Arbeitszeitlage wurde anhand zweier Variablen gemessen. Die dichotom abgefragten Items zur Samstags- und Sonntagsarbeit $(0=$ nein; $1=\mathrm{ja})$ wurden zusammengefasst zur Wochenendarbeit. Diese liegt vor, sofern eines der beiden Einzelitems mit ,ja“ beantwortet wurde. Zusätzlich wurde die dichotom abgefragte Variable Arbeitszeit vor 7 oder nach 19 Uhr $(0=$ nein; $1=\mathrm{ja})$ aufgenommen.

Zwei Formen betrieblicher Veränderungen des unmittelbaren Arbeitsumfelds in den zwei Jahren vor der Befragung wurden erfasst: Umstrukturierungen oder Umorganisationen sowie Stellenabbau oder Entlassungen. Beide Variablen wurden dichotom $(0=$ nein; $1=$ ja $)$ abgefragt.

Herausforderungen Für die psychisch-informatorischen Herausforderungen konnten keine Faktoren eindeutig extrahiert werden. In der Regressionsanalyse werden deshalb die Einzelitems berücksichtigt. Die Items Termin- oder Leistungsdruck, schnelles Arbeiten und Multitasking wurden auf einer vierstufigen Likert-Skala $(1=$ nie; $2=$ selten; $3=$ manchmal; 4=häufig) gemessen. Das Item Nutzung von Informations- und Kommunikationstechnologien (IKT) wurde dichotom $(0=$ nein; $1=$ ja $)$ abgefragt.

\subsubsection{Ressourcen}

Organisationale Ressourcen Für die berufliche Autonomie wurde aus einer Gruppe von sechs Items ein gemeinsamer Faktor (Cronbachs Alpha: 0,70) identifiziert. Der Faktor umfasst zum einen fünf Items, welche auf einer 4-stufigen Likert-Skala ( $1=$ nie; $2=$ selten; $3=$ manchmal; $4=$ häufig) gemessen wurden. Im Einzelnen sind dies: Einfluss auf Pausen, auf Planung bzw. Einteilung sowie auf Menge und Art der Arbeit. Zum anderen umfasst es das Item Einfluss auf Arbeitsbeginn und-ende, welches auf einer 5-stufigen Likert-Skala $(1=$ sehr wenig; $5=$ sehr hoch $)$ gemessen wurde.

Soziale Unterstützung wurde anhand von sechs vierstufig Likert-skalierten Items $(1=$ nie; $2=$ selten; $3=$ manchmal; $4=$ häufig) gemessen. Mit Hilfe einer Faktorenanalyse konnten zwei Faktoren identifiziert werden. Der erste Faktor misst die soziale Unterstïtzung durch Kollegen (Cronbachs Alpha: 0,63) und beinhaltet das Gefühl, Teil einer Gemeinschaft am Arbeitsplatz zu sein sowie das Empfinden guter Zusammenarbeit mit und sozialer Unterstützung von Kollegen. Der zweite Faktor bezieht sich auf direkte Vorgesetzte (Cronbachs Alpha: 0,66) und beinhaltet die Hilfe und Unterstützung, die Möglichkeit der Beteiligung an der Arbeitsgestaltung und -Verbesserung sowie die Häufigkeit einer rücksichtlosen Behandlung.

Das Betriebsklima wurde wiederum mit Hilfe einer Faktorenanalyse in zwei Dimensionen differenziert. Die erste Dimension betrifft die Gerechtigkeit und Anerkennung (Cronbachs Alpha: 0,70) und beinhaltet vier Items. Die Beschäftigten nehmen wahr, dass die Arbeit in ihren Unternehmen gerecht verteilt wird, man seine Meinung offen äußern kann, für geleistete Arbeit Anerkennung erhält und dass die Arbeit angemessene Karrierechancen bietet. Der zweite Faktor misst die Rollenklarheit (Cronbachs Alpha: 0,82 ) und impliziert anhand seiner zwei Items das Wissen darüber, welche Aufgaben in den eigenen Verantwortungsbereich fallen und was bei der Arbeit erwartet wird. Alle Items wurden auf einer fünfstufigen Likert-Skala $(1=$ trifft in nur sehr geringem Maße zu; $5=$ trifft in einem sehr hohen Maße zu) gemessen.

Individuelle Ressourcen Das Bildungsniveau wurde gemäß der internationalen Standardklassifikation (ISCED) dreistufig gemessen und reicht von niedrig über mittel bis hoch. Die berufliche Selbstwirksamkeitserwartung (Cronbachs Alpha: 0,86) wiederum entspricht einem einzeln extrahierten Faktor aus fünf Items, welche auf einer fünfstufigen Likert-Skala $(1=$ trifft überhaupt nicht $\mathrm{zu} ; 5=$ trifft voll und ganz zu) gemessen wurden. Ein Beispiel-Item lautet: 
„Wenn im Beruf unerwartete Situationen auftauchen, weiß ich immer, wie ich mich verhalten soll“".

Drei soziodemografische Merkmale wurden als Kontrollvariablen in die Modelle aufgenommen: das Geschlecht Frau, das Ausüben einer Führungsposition sowie das Alter in Jahren (15-67).

\section{Ergebnisse}

Zunächst wird ein deskriptiver Vergleich von Anforderungen, Ressourcen und Arbeitsfähigkeit in den beiden Berufsgruppen zwischen 2015 und 2017 durchgeführt. Im Anschluss folgt ein Vergleich der beiden Berufsgruppen mit dem Durchschnitt aller anderen Berufsgruppen. Ausgangspunkt für diesen Vergleich ist das Jahr 2015, da hier ausreichend hohe Fallzahlen zur Verfügung stehen. Zudem sind, wie sich zeigen wird, die Veränderungen zwischen den Jahren 2015 und 2017 nicht grundlegend. Eine hohe Fallzahl wird vor allem für die im Anschluss folgenden multiplen linearen Regressionsmodelle benötigt, die deshalb ausschließlich für das Jahr 2015 vorgenommen wurden. Diese Modelle ermöglichen es, den Einfluss von beobachteten Drittvariablen zu kontrollieren. Die Ergebnisse können dennoch nur unter Vorbehalt kausal interpretiert werden. Festgestellt werden kann bspw. lediglich ein Zusammenhang geringerer Arbeitsfähigkeit mit geringeren Ressourcen. $\mathrm{Ob}$ sinkende Ressourcen zu einer geringeren Arbeitsfähigkeit führen oder ob Beschäftigte mit geringer Arbeitsfähigkeit lediglich Arbeitsstellen mit geringen organisationalen Ressourcen finden, kann hier nicht abschließend beurteilt werden. Die Schlussfolgerungen im Anschluss an die folgenden Analysen sind deshalb als Hinweise auf bestehende Zusammenhänge zu verstehen, die einer weiteren Prüfung bedürfen.

\subsection{Veränderungen in Lagerwirtschaft und Postberufen zwischen 2015 und 2017}

Ein Vergleich innerhalb der Lagerwirtschaft zwischen den Jahren 2015 und 2017 (s. Tab. 5 im Anhang) macht deutlich, dass sich im Verlauf der zwei Jahre hinsichtlich der Anforderungen auf Ebene der Stressoren lediglich die Unterbrechungen durch Kollegen bedeutsam zugenommen haben. Auf Ebene der Herausforderungen konnte eine signifikant vermehrte gleichzeitige Erledigung von Arbeitsabläufen im Jahr 2017 zum Jahr 2015 ausgemacht werden. Während die organisationalen Ressourcen sich zwischen den Jahren nicht relevant unterscheiden, ist dies für die individuellen Ressourcen in Form des Bildungsniveaus und der Selbstwirksamkeit der Fall. Die hier hervorgehobenen Unterschiede werden jedoch alle durch sehr niedrige Effektstärken relativiert. Der signifikante Anstieg des durchschnittlichen Al- ters zwischen 2015 und 2017 von etwa 45 auf 48 Jahre ist überwiegend dem Umstand geschuldet, dass es sich um ein Paneldesign handelt und zu einem erheblichen Anteil dieselben Personen im Abstand von zwei Jahren befragt wurden.

Für Beschäftigte im Postberuf hat sich hinsichtlich der Stressoren die subjektiv wahrgenommene Belastung durch Hitze und Licht zwischen den Jahren 2015 und 2017 in geringem Maße signifikant verringert. Zudem kann auch bei dieser Beschäftigtengruppe eine geringfügige Verbesserung der Ausstattung mit individuellen Ressourcen in Form einer erhöhten Selbstwirksamkeit aufgezeigt werden. Diese Ergebnisse sind jedoch aufgrund der niedrige Effektstärken zwischen 0,13 und 0,15 wie bereits bei der Gruppe der Lagerwirtschaft mit Zurückhaltung zu interpretieren.

Alle anderen in unserem Modell berücksichtigten Variablen weisen für Beschäftigte in beiden Berufsgruppen keinerlei signifikante Unterschiede zwischen den Jahren 2015 und 2017 auf. Um hier Differenzen wahrnehmen zu können, bedarf es vermutlich einer längeren Zeitspanne. Da die Daten für beide Zeiträume kaum signifikante Unterschiede aufweisen und der Datensatz für das Jahr 2015 sowohl eine größere Stichprobe für die einzelnen Berufsgruppen als auch weitere Variablen aufweist, welche im Jahr 2017 nicht wieder erhoben wurden, werden sich nachfolgende Analysen auf den Datensatz der Arbeitszeitbefragung 2015 beziehen.

\subsection{Vergleich von Lagerwirtschaft, Postberufen und allen anderen Berufsgruppen}

Der Vergleich der einzelnen Berufsgruppen mit dem Subdatensatz der restlichen Beschäftigten als Vergleichsgruppe (s. Tab. 2) verdeutlicht zunächst, dass sowohl Beschäftigte in der Lagerwirtschaft als auch in den Postberufen über schlechtere Arbeitsfähigkeit berichten als Beschäftigte in anderen Berufsgruppen. Allerdings ist lediglich der Unterschied zwischen Beschäftigten in der Lagerwirtschaft und den restlichen Befragten signifikant. Darüber hinaus sind beide Berufsgruppen häufiger den Stressoren physischer Arbeitsbelastungen ausgesetzt, z. B. durch Arbeiten im Stehen oder Arbeitsumfeldeinflüsse wie Hitze. Auch atypische Arbeitszeiten wie Wochenendarbeit werden von beiden Berufsgruppen wesentlich häufiger angegeben als von den restlichen Beschäftigten. Unter den Herausforderungen ist vor allem geringere Nutzung moderner Informations- und Kommunikationstechnologien hervorzuheben. Hinsichtlich der organisationalen Ressourcen gilt für Beschäftigte in der Intralogistik und den Postberufen im Vergleich zu den restlichen Beschäftigten: ihr Handlungsspielraum ist geringer und sie treffen seltener auf ein Betriebsklima der Anerkennung. Zudem ist das Bildungsniveau in beiden Berufsgruppen bedeutsam geringer. 
Tab. 2 Vergleich der Berufsgruppen mit den restlichen Beschäftigten sowie miteinander, 2015

Table 2 Comparison of the occupational groups with the remaining employees and with each other, 2015

\begin{tabular}{|c|c|c|c|c|c|c|c|}
\hline \multicolumn{2}{|l|}{ Item } & \multicolumn{3}{|c|}{ Mittelwerte } & \multicolumn{3}{|l|}{ Effektstärke $|\mathrm{r}|$} \\
\hline Bezeichnung & Min - Max & Rest & Lager & Post & Rest vs. Lager & Rest vs. Post & Lager vs. Post \\
\hline Bewertung eigener Arbeitsfähigkeit & $0-10$ & 7,82 & 7,21 & 7,49 & $0,09 * *$ & 0,05 & 0,03 \\
\hline \multicolumn{8}{|l|}{ Anforderungen: Stressoren } \\
\hline Im Stehen arbeiten & $1-4$ & 2,91 & 3,70 & 3,68 & $0,37 * * *$ & $0,24 * * *$ & 0,00 \\
\hline Mit schweren Lasten arbeiten & $1-4$ & 1,99 & 2,90 & 3,36 & $0,35 * * *$ & $0,34 * * *$ & $0,19 * * *$ \\
\hline $\begin{array}{l}\text { In kniender Stellung, über Kopf } \\
\text { arbeiten }\end{array}$ & $1-4$ & 1,80 & 2,36 & 2,39 & $0,25 * * *$ & $0,19 * * *$ & 0,01 \\
\hline Unter Hitze arbeiten & $1-4$ & 2,04 & 2,64 & 3,65 & $0,23 * * *$ & $0,38 * * *$ & $0,38 * * *$ \\
\hline Bei grellem Licht arbeiten & $1-4$ & 1,68 & 1,94 & 2,52 & $0,10 * * *$ & $0,25 * * *$ & $0,24 * * *$ \\
\hline Unter Lärm arbeiten & $1-4$ & 2,14 & 2,59 & 2,33 & $0,17 * * *$ & $0,06^{+}$ & $0,09^{+}$ \\
\hline Unterbrechung durch Kollegen & $1-4$ & 3,27 & 3,04 & 2,62 & $0,10^{* * *}$ & $0,23 * * *$ & $0,19 * * *$ \\
\hline Wochenendarbeit ${ }^{a}$ & $0-1$ & 0,58 & 0,66 & 0,87 & $0,08 * a$ & $0,24 * * * a$ & $0,20 * * * a$ \\
\hline Arbeitszeit außerhalb 7 bis 19 Uhr ${ }^{a}$ & $0-1$ & 0,15 & 0,36 & 0,28 & $0,24 * * * a$ & $0,13 * * * a$ & $0,08^{a}$ \\
\hline $\begin{array}{l}\text { Umstrukturierung in den letzten } 2 \\
\text { Jahren }^{\mathrm{a}}\end{array}$ & $0-1$ & 0,37 & 0,41 & 0,55 & $0,04^{\mathrm{a}}$ & $0,14 * * * a$ & $0,12 * * a$ \\
\hline $\begin{array}{l}\text { Stellenabbau in den letzten } 2 \\
\text { Jahren }^{\mathrm{a}}\end{array}$ & $0-1$ & 0,30 & 0,33 & 0,39 & $0,03^{\mathrm{a}}$ & $0,08^{+a}$ & $0,06^{\mathrm{a}}$ \\
\hline \multicolumn{8}{|l|}{ Anforderungen: Herausforderungen } \\
\hline $\begin{array}{l}\text { Starker Termin- oder Leistungs- } \\
\text { druck }\end{array}$ & $1-4$ & 3,34 & 3,12 & 3,31 & $0,08^{*}$ & 0,02 & $0,09^{+}$ \\
\hline Sehr schnelles Arbeiten & $1-4$ & 3,27 & 3,32 & 3,56 & $0,05^{+}$ & $0,14 * * *$ & $0,13 * * *$ \\
\hline $\begin{array}{l}\text { Gleichzeitige Erledigung von Ar- } \\
\text { beitsabläufen }\end{array}$ & $1-4$ & 3,56 & 3,09 & 3,14 & $0,24 * * *$ & $0,14 * * *$ & 0,03 \\
\hline Nutzung moderner IKT ${ }^{a}$ & $0-1$ & 0,77 & 0,42 & 0,26 & $0,35 * * * a$ & $0,42 * * * a$ & $0,14 * * * a$ \\
\hline \multicolumn{8}{|l|}{ Ressourcen: Organisational } \\
\hline $\begin{array}{l}\text { Einfluss auf Arbeitsbeginn und } \\
\text {-ende }\end{array}$ & $1-5$ & 3,11 & 2,00 & 2,11 & $0,32 * * *$ & $0,20 * * *$ & 0,05 \\
\hline $\begin{array}{l}\text { Arbeitsdurchführung vorgeschrie- } \\
\text { ben }\end{array}$ & $1-4$ & 2,50 & 2,97 & 3,11 & $0,19 * * *$ & $0,17 * * *$ & 0,06 \\
\hline $\begin{array}{l}\text { Eigene Arbeit selbst planen und } \\
\text { einteilen }\end{array}$ & $1-4$ & 3,64 & 3,00 & 3,08 & $0,30 * * *$ & $0,18 * * *$ & 0,04 \\
\hline Einfluss auf Arbeitsmenge & $1-4$ & 2,73 & 2,31 & 1,78 & $0,16 * * *$ & $0,26 * * *$ & $0,21 * * *$ \\
\hline Eigene Entscheidung über Pausen & $1-4$ & 3,27 & 2,45 & 3,23 & $0,29 * * *$ & 0,01 & $0,25^{* * *}$ \\
\hline Einfluss auf Aufgabenart & $1-4$ & 2,83 & 2,31 & 1,87 & $0,21 * * *$ & $0,27 * * *$ & $0,18 * * *$ \\
\hline $\begin{array}{l}\text { Teil der Gemeinschaft am Arbeits- } \\
\text { platz. }\end{array}$ & $1-4$ & 3,56 & 3,27 & 3,25 & $0,14 * * *$ & $0,11 * * *$ & 0,02 \\
\hline Gute Zusammenarbeit mit Kollegen & $1-4$ & 3,80 & 3,71 & 3,63 & 0,05 & $0,08 * *$ & 0,07 \\
\hline Unterstützung von Kollegen & $1-4$ & 3,67 & 3,55 & 3,39 & 0,04 & $0,10 * * *$ & $0,08^{+}$ \\
\hline $\begin{array}{l}\text { Unterstützung von Führungskraft } \\
\text { (FK) }\end{array}$ & $1-4$ & 3,11 & 2,95 & 2,88 & $0,06^{+}$ & $0,07 *$ & 0,04 \\
\hline $\begin{array}{l}\text { FK gibt Möglichkeit zur Beteili- } \\
\text { gung }\end{array}$ & $1-4$ & 3,17 & 2,84 & 2,51 & $0,14 * * *$ & $0,20 * * *$ & $0,13 * * *$ \\
\hline $\begin{array}{l}\text { Rücksichtslose Behandlung durch } \\
\text { FK }\end{array}$ & $1-4$ & 1,56 & 1,72 & 1,64 & $0,07 *$ & 0,04 & 0,02 \\
\hline Gerechte Verteilung der Arbeit & $1-5$ & 3,53 & 3,33 & 3,34 & $0,06^{+}$ & 0,04 & 0,00 \\
\hline $\begin{array}{l}\text { Offene Äußerung der Meinung } \\
\text { möglich }\end{array}$ & $1-5$ & 3,97 & 3,53 & 3,48 & $0,15 * * *$ & $0,11 * * *$ & 0,02 \\
\hline $\begin{array}{l}\text { Angemessene Anerkennung der } \\
\text { Arbeit }\end{array}$ & $1-5$ & 3,79 & 3,41 & 3,36 & $0,13 * * *$ & $0,11 * * *$ & 0,02 \\
\hline Angemessene Karrierechancen & $1-5$ & 2,98 & 2,39 & 2,23 & $0,21 * * *$ & $0,18 * * *$ & 0,05 \\
\hline $\begin{array}{l}\text { Transparenz über Verantwortungs- } \\
\text { bereich }\end{array}$ & $1-5$ & 4,52 & 4,42 & 4,63 & 0,01 & $0,08 * *$ & $0,12 * *$ \\
\hline Transparenz über Erwartung & $1-5$ & 4,60 & 4,61 & 4,71 & $0,06^{+}$ & $0,09 * * *$ & $0,08^{+}$ \\
\hline
\end{tabular}


Tab. 2 (Fortsetzung)

Table 2 (Continued)

\begin{tabular}{|c|c|c|c|c|c|c|c|}
\hline \multicolumn{2}{|l|}{ Item } & \multicolumn{3}{|c|}{ Mittelwerte } & \multicolumn{3}{|l|}{ Effektstärke $|\mathrm{r}|$} \\
\hline Bezeichnung & Min - Max & Rest & Lager & Post & Rest vs. Lager & Rest vs. Post & Lager vs. Post \\
\hline \multicolumn{8}{|l|}{ Ressourcen: Individuell } \\
\hline Bildungsniveau & $1-3$ & 2,44 & 2,02 & 2,04 & $0,35 * * *$ & $0,24 * * *$ & 0,02 \\
\hline $\begin{array}{l}\text { Guter Umgang mit unerwarteten } \\
\text { Situationen }\end{array}$ & $1-5$ & 3,82 & 3,68 & 3,73 & 0,02 & 0,01 & 0,02 \\
\hline $\begin{array}{l}\text { Gelassenheit bei beruflichen } \\
\text { Schwierigkeiten }\end{array}$ & $1-5$ & 3,98 & 3,87 & 4,00 & 0,00 & 0,04 & 0,05 \\
\hline $\begin{array}{l}\text { Ideen zur Problemlösung bei der } \\
\text { Arbeit }\end{array}$ & $1-5$ & 3,94 & 3,77 & 3,83 & $0,06^{+}$ & 0,01 & 0,04 \\
\hline $\begin{array}{l}\text { Den Anforderungen im Beruf ge- } \\
\text { wachsen }\end{array}$ & $1-5$ & 4,27 & 4,10 & 4,16 & 0,04 & 0,01 & 0,04 \\
\hline $\begin{array}{l}\text { Gut auf berufliche Zukunft vorberei- } \\
\text { tet }\end{array}$ & $1-5$ & 4,19 & 3,74 & 3,96 & $0,15 * * *$ & 0,05 & 0,07 \\
\hline \multicolumn{8}{|l|}{ Soziodemografie/Kontrollvariablen } \\
\hline Alter & $16-67$ & 45,6 & 45,1 & 48,0 & 0,02 & $0,06^{+}$ & $0,10^{*}$ \\
\hline Weibliches Geschlecht ${ }^{a}$ & $0-1$ & 0,53 & 0,36 & 0,52 & $0,17 * * * a$ & $0,01^{a}$ & $0,15 * * * a$ \\
\hline Führungskraft ${ }^{\mathrm{a}}$ & $0-1$ & 0,37 & 0,23 & 0,17 & $0,14 * * * a$ & $0,17 * * * a$ & $0,07^{\mathrm{a}}$ \\
\hline
\end{tabular}

Hinweis: Mann-Whitney-U-Tests mit den jeweiligen Subgruppen Lagerwirtschaft $(n=348)$ und Post- und Zustelldienste $(n=121)$ sowie einem Subdatensatz aus allen restlichen Beschäftigten $(n=500)$ für das Jahr 2015

Berechnung von $\mathrm{r}$ folgt der Formel $\mathrm{Z} / \sqrt{(\mathrm{N}}_{\mathrm{x}}+\mathrm{N}_{\mathrm{y}}$ ) (Pallant 2007). $* * * \leq 0,001 ; * * \leq 0,01 ; * \leq 0,05 ;{ }^{+} \leq 0,1$. Gebündelt kursiv/steil markierte Items markieren ein gemeinsames Konstrukt (z. B. berufliche Autonomie)

$M W$ Mittelwert (ungewichtet), $r$ Effektstärke

${ }^{\mathrm{a}}=\mathrm{Chi}^{2}$-Test für dichotome Variablen mit Cramers-V als Effektstärke

Diesen Gemeinsamkeiten stehen allerdings einige Unterschiede im Vergleich der einzelnen Berufsgruppen mit den restlichen Beschäftigten gegenüber. So berichten nur Erwerbstätige in Postberufen signifikant häufiger von Umstrukturierungen und Stellenabbau in den Betrieben. Demgegenüber verfügen sie im Gegensatz zu Beschäftigten in der Intralogistik häufiger über ein Betriebsklima der Rollenklarheit sowie der guten Zusammenarbeit mit und Unterstützung von Kollegen.

Neben den Vergleichen mit den restlichen Erwerbstätigen können darüber hinaus die Lagerwirtschaftsberufe direkt mit den Postberufen verglichen werden. Dies liefert erste bivariate Erkenntnisse über Unterschiede der Arbeitssituation der jeweiligen Beschäftigtengruppen: Mit Blick auf die Stressoren sind Beschäftigte in der Lagerwirtschaft weniger von physischen Arbeits- sowie Arbeitsumfeldbelastungen betroffen. Zudem arbeiten sie seltener am Wochenende und haben in den letzten zwei Jahren weniger Umstrukturierungsmaßnahmen erfahren. Hinsichtlich der Herausforderungen sind Postbeschäftigte häufiger von schnellem Arbeiten betroffen, während Intralogistiker häufiger moderne IKT einsetzen. Bezüglich organisationaler Ressourcen in Form betrieblicher Autonomie haben Intralogistiker einen stärkeren Einfluss auf die Arbeitsmenge und -art, können aber seltener eigene Entscheidungen über Pausen treffen. Unterstützung durch Kollegen und eine durch die Führungskraft bereitgestellte Möglichkeit zur
Beteiligung werden vermehrt von den Beschäftigten in der Lagerwirtschaft wahrgenommen. Dahingegen berichten Postbeschäftigte häufiger von einer Transparenz über den Verantwortungsbereich und der Erwartungshaltung. In den Postberufen lassen sich ferner geringfügig ältere sowie weibliche Beschäftigte finden.

\subsection{Erklärung berufsgruppenspezifischer Unterschiede in der Arbeitsfähigkeit}

Im Folgenden werden lineare Regressionsmodelle der Arbeitsfähigkeit dargestellt, welche alle Anforderungen und Ressourcen beinhalten. Das Gesamtmodell (Tab. 3, Spalte „Gesamt") umfasst die Stichprobe aller Berufe. Als zentrale unabhängige Variablen gehen hier zusätzlich die beiden Berufsgruppen Lagerwirtschaft und Post- und Zustelldienste in das Modell ein. Die zugehörigen Koeffizienten geben Unterschiede in der Arbeitsfähigkeit bei den beiden jeweiligen Berufen im Vergleich zum Rest der Berufe wieder, wenn Unterschiede in den Arbeitsbedingungen berücksichtigt werden. Folglich erklären diese Unterschiede einen Teil der berufsgruppenspezifischen Unterschiede in der Arbeitsfähigkeit. In zusätzlichen Einzelmodellen wurde überprüft, welche jeweiligen einzelnen Arbeitsbedingungen den größten Erklärungsbeitrag leisten.

Blickt man zunächst auf die Koeffizienten der Anforderungen und Ressourcen im Gesamtmodell, so wird deutlich, 
Tab. 3 Regressionsmodelle mit Arbeitsfähigkeit als abhängiger Variable für alle Beschäftigte sowie beide Berufsgruppen separat, 2015

Table 3 Regression models with work ability as a dependent variable for all employees and both occupational groups separately, 2015

\begin{tabular}{|c|c|c|c|c|}
\hline Variable & Variable & Gesamt & Lagerwirtschaft & Postberufe \\
\hline \multirow[t]{2}{*}{ Berufsgruppe } & Lagerwirtschaft & $-0,268 * *$ & - & - \\
\hline & Post- und Zustelldienste & 0,150 & & \\
\hline \multicolumn{5}{|l|}{ Anforderungen } \\
\hline \multirow[t]{7}{*}{ Stressoren } & Physische Arbeitsbelastungen & $-0,080 * * *$ & $-0,033$ & $-0,156$ \\
\hline & Arbeitsumfeldbelastungen & $-0,070 * * *$ & $-0,033$ & $0,260 *$ \\
\hline & $\begin{array}{l}\text { Unterbrechung durch Kolle- } \\
\text { gen }\end{array}$ & $-0,057 * *$ & $-0,059$ & $-0,238^{*}$ \\
\hline & Arbeiten am Wochenende & 0,036 & 0,017 & $-0,172$ \\
\hline & $\begin{array}{l}\text { Arbeitszeit außerhalb } 7 \text { und } \\
19 \text { Uhr }\end{array}$ & $-0,091 *$ & $-0,079$ & $-0,094$ \\
\hline & Umstrukturierung & $-0,060^{*}$ & $-0,039$ & 0,092 \\
\hline & Stellenabbau & $-0,062^{+}$ & $-0,064$ & 0,114 \\
\hline \multirow[t]{4}{*}{$\begin{array}{l}\text { Heraus- } \\
\text { forderungen }\end{array}$} & $\begin{array}{l}\text { Starker Termin- und Leis- } \\
\text { tungsdruck }\end{array}$ & $-0,088 * * *$ & $-0,127^{+}$ & $-0,264^{+}$ \\
\hline & Schnelles Arbeiten & $-0,018$ & $0,136^{+}$ & 0,099 \\
\hline & Multitasking & 0,007 & 0,024 & $-0,114$ \\
\hline & Nutzung von IKT & $-0,041$ & $-0,161 * *$ & $-0,039$ \\
\hline \multicolumn{5}{|l|}{ Ressourcen } \\
\hline \multirow[t]{5}{*}{ Organisational } & Berufliche Autonomie & $0,035 * * *$ & 0,095 & $-0,098$ \\
\hline & $\begin{array}{l}\text { Soziale Unterstützung durch } \\
\text { Kollegen }\end{array}$ & $0,118 * * *$ & $0,128 *$ & 0,118 \\
\hline & $\begin{array}{l}\text { Soziale Unterstützung durch } \\
\text { Vorgesetzte }\end{array}$ & $0,126^{* * * *}$ & $-0,006$ & 0,117 \\
\hline & Betriebsklima: Anerkennung & $0,221 * * *$ & 0,076 & $-0,089$ \\
\hline & Betriebsklima: Rollenklarheit & $0,073 * * *$ & $0,122 *$ & 0,109 \\
\hline \multirow[t]{2}{*}{ Individuell } & Bildungsniveau & $0,070^{*}$ & 0,030 & $-0,018$ \\
\hline & Selbstwirksamkeit & $0,268 * * *$ & 0,090 & $0,250 *$ \\
\hline \multicolumn{5}{|l|}{ Kontrollvariablen } \\
\hline \multirow[t]{3}{*}{ Soziodemografie } & Alter & $-0,015^{* * *}$ & $-0,065$ & $-0,169$ \\
\hline & Geschlecht (weiblich) & $-0,020$ & $-0,061$ & 0,075 \\
\hline & Führungskraft & $0,066^{*}$ & $-0,038$ & $0,215^{*}$ \\
\hline$N$ & & 16.659 & 317 & 106 \\
\hline Korrigiertes $R^{2}$ & & 0,128 & 0,108 & 0,155 \\
\hline
\end{tabular}

Hinweis: Für die beiden Berufsgruppen sind standardisierte Koeffizienten ausgegeben. $* * * \leq 0,001 ; * * \leq 0,01$; $* \leq 0,05 ;{ }^{+} \leq 0,1$ dass sich die Annahmen des erweiterten Anforderungs-Ressourcen-Modells über die Zusammenhänge mit der Arbeitsfähigkeit (vgl. Abb. 1) größtenteils bestätigen. Die vorab als Stressoren angenommenen Variablen und Faktoren weisen fast durchweg signifikant negative, die Ressourcen allesamt signifikant positive Vorzeichen auf. Die angenommene Ambivalenz der Herausforderungen zeigt sich im Gesamtmodell insofern, als dass die Werte von drei der vier Variablen sich nicht signifikant unterscheiden. Allerdings kann der starke Termin- und Leistungsdruck eindeutig als Stressor identifiziert werden, auch wenn man die Modelle der einzelnen Berufsgruppen hinzuzieht.

Der Blick auf den signifikant negativen Koeffizienten der Lagerwirtschaft im Gesamtmodell macht deutlich, dass die geringere Arbeitsfähigkeit dort nicht vollständig durch Unterschiede in den Anforderungen und Ressourcen erklärt werden kann. Beschäftigte in der Lagerwirtschaft haben somit eine geringere Arbeitsfähigkeit als die restlichen Beschäftigten in der Stichprobe, auch wenn für alle im Modell enthaltenen Variablen kontrolliert wird. Demnach gibt es in der Lagerwirtschaft Einflüsse auf die Arbeitsfähigkeit, die nicht durch die im Modell enthaltenen Variablen erklärt werden können. Im Gegensatz zur Lagerwirtschaft ist der Effekt der Postberufe auf die Arbeitsfähigkeit zwar leicht positiv, aber nicht signifikant. Der bereits oben festgestellte signifikante Unterschied zwischen der Arbeitsfähigkeit in den Postberufen und den restlichen Beschäftigten lässt sich somit durch die im Modell enthaltenen Variablen erklären. 


\subsection{Einflussfaktoren der Arbeitsfähigkeit nach Berufsgruppe}

Die zuvor durchgeführten Analysen untersuchten Unterschiede in der Arbeitsfähigkeit bei den Beschäftigtengruppen der Lagerwirtschafts- bzw. Postberufe im Vergleich zu allen anderen Erwerbstätigen und beinhalteten die gesamte Stichprobe. Abschließend soll analysiert werden, welche Faktoren spezifisch für die Arbeitsfähigkeit der Beschäftigten innerhalb der beiden jeweiligen Berufsgruppen relevant sind und ob diese sich zwischen den Gruppen unterscheiden.

Im Modell für die Lagerwirtschaft (s. Tab. 3, Spalte „Lagerwirtschaft") weisen lediglich vereinzelte Anforderungen und Ressourcen signifikante Effekte auf. So stehen die Ressourcen der sozialen Unterstützung durch Kollegen sowie eines durch eindeutige Rollenzuordnung gekennzeichneten Betriebsklimas in Zusammenhang mit einer erhöhten Arbeitsfähigkeit. Dies gilt ebenso für die Variable „schnelles Arbeiten“, die sich damit als eine positiv wirksame Herausforderung erweist. Demgegenüber geht die Nutzung moderner Informations- und Kommunikationsmittel mit einer Reduzierung der Arbeitsfähigkeit einher. Diese lässt sich somit für die Lagerwirtschaft nicht als Herausforderung, sondern als Stressor sehen. Die Nutzung moderner IKT leistet zudem innerhalb des Modells den höchsten relativen Beitrag aller Variablen zur Erklärung der Arbeitsfähigkeit.

Das Modell für die Postberufe (s. Tab. 3, Spalte „Postberufe") zeigt zunächst einen positiven Zusammenhang der ungünstigen physischen Arbeitsumfeldbedingungen - Arbeiten bei grellem Licht, Lärm und Hitze - mit der Arbeitsfähigkeit. Dies ist überraschend, da diese im empirischen Regressionsmodell mit allen anderen Erwerbstätigen negativ mit der Arbeitsfähigkeit assoziiert sind (Tab. 3, Spalte "Gesamt") und auch theoretisch als Stressoren gewertet wurden. Negativ signifikant ist erwartungsgemäß aber die Unterbrechung durch Kollegen. Wie schon bei der Lagerwirtschaft ist der starke Termin- und Leistungsdruck signifikant negativ und damit als Stressor zu werten. Diese Variable leistet in den Postberufen sogar den höchsten relativen Beitrag zur Erklärung der Arbeitsfähigkeit von Beschäftigten. Dagegen stehen die Ressource einer höheren Selbstwirksamkeit sowie das Ausüben einer Führungsposition in einem positiven Zusammenhang mit der abhängigen Variablen. Die wenigen Führungskräfte in den Postberufen unterscheiden sich somit grundlegend von den Beschäftigten ohne Führungsverantwortung.

Hinsichtlich der Modellgüte weist das Modell für die Postberufe mit 15,5\% aufgeklärter Varianz geringfügig bessere Werte auf als das Modell der Lagerwirtschaft (10,8\% aufgeklärte Varianz). Beide Kennziffern deuten jedoch darauf hin, dass es weitere relevante Faktoren zur Erklärung der Arbeitsfähigkeit von Beschäftigten in der Lagerwirt- schaft und in Postberufen gibt, die nicht in unserem Modell berücksichtigt wurden.

\section{Diskussion und Fazit}

Die vorliegende Untersuchung ging anhand der BAuA-Arbeitszeitbefragung für die Beschäftigten in der Lagerwirtschaft und den Post- und Zustelldiensten der Frage nach, in welchem Zusammenhang deren Arbeitsfähigkeit mit den dortigen Arbeitsbedingungen steht. Welche Anforderungen wirken als besonders prekäre Stressoren, welche eher ambivalent als Herausforderungen? Gibt es organisationale Ressourcen, welche die Arbeitsfähigkeit fördern? Die Beantwortung dieser Fragen liefert konkrete Ansatzpunkte für eine menschengerechte Arbeitsgestaltung.

Zunächst zeigt sich für alle Berufe, dass sich weder die Arbeitsbedingungen noch die Arbeitsfähigkeit zwischen den Jahren 2015 und 2017 im Großen und Ganzen signifikant verändert haben. Im Querschnittvergleich des Jahres 2015 - aus Fallzahlengründen wurde die jüngere Welle nicht berücksichtigt - beurteilen beide hier untersuchten Berufsgruppen ihre Arbeitsfähigkeit schlechter als der Durchschnitt aller anderen Erwerbstätigen, auch wenn nur der Unterschied in der Lagerwirtschaft signifikant ist. Zudem sind sie im Wesentlichen häufiger physischen Stressoren ausgesetzt, haben geringere organisationale Ressourcen, wie Anerkennung sowie soziale Unterstützung durch Kollegen oder Führungskräfte oder beruflichen Entscheidungsspielraum, sowie ein geringeres Bildungsniveau als individuelle Ressource.

Der deskriptive Vergleich zwischen den beiden untersuchten Berufsgruppen zeigt einige Besonderheiten auf. Die hier erfassten Stressoren der physischen Arbeitsbelastungen sind für die in den Postberufen Beschäftigten im Vergleich zur Lagerwirtschaft noch einmal höher, dagegen treten Unterbrechungen durch Kollegen seltener auf. Postbeschäftigte weisen auch eine geringere Ressource beruflicher Autonomie bezüglich der Arbeitsmenge und Aufgabenart auf, wohingegen sie im Vergleich zu Beschäftigten der Intralogistik freier über den Zeitpunkt der Pausen bestimmen können. Sie erhalten zudem seltener soziale Unterstützung durch Kollegen sowie durch Führungskräfte in Form von Beteiligungsmöglichkeiten. Das Betriebsklima in der Dimension der Rollenklarheit, das heißt der Transparenz hinsichtlich der Erwartungen und des Verantwortungsbereichs, ist hingegen besser. Auch bei Arbeitsbedingungen, die zunächst theoretisch als Herausforderungen gewertet wurden, zeigen sich Unterschiede. Die in den Berufen der Lagerwirtschaft Beschäftigten weisen einen geringeren Terminund Leistungsdruck auf, müssen seltener schnell arbeiten und nutzen häufiger moderne Informations- und Kommunikationstechnologie. Darüber hinaus leisten Intralogistiker 
Tab. 4 Zentrale Ergebnisse aus den Analysen und daraus abgeleitete Ansatzpunkte für eine menschengerechte Arbeitsgestaltung Table 4 Main results and approaches for preventive human-centred measuress

\begin{tabular}{|c|c|c|}
\hline Arbeitsbedingung & $\begin{array}{l}\text { Betroffene Berufs- } \\
\text { gruppe }\end{array}$ & Ansatzpunkte für präventive Maßnahmen \\
\hline \multicolumn{3}{|c|}{ Arbeitsbedingungen mit bedeutsamen negativen Zusammenhang zur Arbeitsfähigkeit } \\
\hline Starker Termin- und Leistungsdruck & $\begin{array}{l}\text { Postberufe } \\
\text { Lagerwirtschaft }\end{array}$ & Verbesserung des Arbeitsflusses, ohne die Intensität zu erhöhen \\
\hline Unterbrechung durch Kollegen & Postberufe & \\
\hline $\begin{array}{l}\text { Nutzung moderner Informations- und } \\
\text { Kommunikationstechnologien }\end{array}$ & Lagerwirtschaft & $\begin{array}{l}\text { Menschengerechte Gestaltung der technischen Infrastruktur unter Berück- } \\
\text { sichtigung der Nutzerakzeptanz und des Anwendungszwecks }\end{array}$ \\
\hline \multicolumn{3}{|c|}{ Arbeitsbedingungen mit bedeutsamen positiven Zusammenhang zur Arbeitsfähigkeit } \\
\hline $\begin{array}{l}\text { Soziale Unterstützung durch Kollegen } \\
\text { Rolle der Führungskraft } \\
\text { Betriebsklima: Rolle }\end{array}$ & $\begin{array}{l}\text { Lagerwirtschaft } \\
\text { Postberufe } \\
\text { Lagerwirtschaft }\end{array}$ & $\begin{array}{l}\text { Intensivere Betonung des sozialen Umfeldes im Arbeitskontext in Form der } \\
\text { Unterstützung durch Kollegen und Führungskräfte sowie des allgemeinen } \\
\text { Betriebsklimas }\end{array}$ \\
\hline
\end{tabular}

weniger Wochenendarbeit und sind seltener von Umstrukturierungen des unmittelbaren Arbeitsumfeldes betroffen. Die individuelle Ausstattung mit Ressourcen in Form von Bildung und Selbstwirksamkeit hingegen unterscheidet sich nicht signifikant.

Regressionsanalysen für alle Beschäftigten sowie separat für beide Berufsgruppen zeigen schließlich vergleichend auf, welche Arbeitsbedingungen signifikant zur Erklärung der Arbeitsfähigkeit der Beschäftigten beitragen. Ein solcher Vergleich der Wirkung von Anforderungen und Ressourcen ermöglicht es, im Sinne eines „Benchmarking“, Restriktionen und „best-practice“-Möglichkeiten der betrieblichen Arbeitsgestaltung im Rahmen eines jeweiligen Berufsfeldes aufzudecken. Zunächst kann mit Blick auf die Arbeitsintensität festgestellt werden, dass es sinnvoll ist, zwischen Stressoren und Herausforderungen zu unterscheiden. So ist schnelles Arbeiten als solches für beide Berufsgruppen positiv mit der Arbeitsfähigkeit assoziiert und kann somit als Herausforderung gewertet werden. Erst wenn ein starker Termin- und Leistungsdruck wahrgenommen wird, besteht Stress, der sich - als einzige Arbeitsbedingung für beide Berufsgruppen - in einer signifikant geringeren Arbeitsfähigkeit ausdrückt. Dieser Befund widerspricht nicht unbedingt dem in der Literatur dokumentierten ambivalenten Charakter des Zeitdrucks, insofern dort Zusammenhänge mit Arbeitsengagement oder allgemeinem Wohlbefinden untersucht wurden (Crawford et al. 2010; Widmer et al. 2012). So stellen Baethge et al. (2018) fest, dass Termindruck nur kurzfristig zu höherem Engagement führt. Langfristig scheinen sich die negativen Auswirkungen auf die Arbeitsfähigkeit durchzusetzen. Die Nutzung von Informations- und Kommunikationstechnologien, oftmals als Herausforderung gewertet, kann hingegen (ausschließlich) in den Lagerwirtschaftsberufen als Stressor identifiziert werden (für die Transportlogistik siehe Schlüter et al. 2018). Den in der Lagerwirtschaft Beschäftigten kommen aber die Ressourcen einer sozialen Unterstützung durch Kollegen sowie eines Klimas der Rollenklarheit zugute. Für Beschäf- tigte der Postberufe wirken die Unterbrechung durch Kollegen und das Arbeiten am Wochenende als Stressoren. Hingegen gehen die individuelle Ressource der Selbstwirksamkeit, aber auch die Besetzung einer Führungsposition mit einer erhöhten Arbeitsfähigkeit einher. Hinter dem letzten Befund, so lässt sich vermuten, können sich nicht erfasste Persönlichkeitseigenschaften, aber auch der soziale Status sowie die besonderen Arbeitsbedingungen von Führungskräften verbergen (Thomson et al. 2020). Der Befund einer erhöhten Arbeitsfähigkeit bei stärkeren Belastungen durch das Arbeitsumfeld ist auf den ersten Blick irritierend. Hier wird ein umgekehrter Kausaleffekt vermutet: Die Arbeit in einem ungünstigen Arbeitsumfeld setzt eine hohe Arbeitsfähigkeit voraus.

Die hier vergleichend dargestellten Gemeinsamkeiten und Unterschiede von Beschäftigten in der Lagerwirtschaft sowie den Post- und Zustelldiensten hinsichtlich der Stressoren, Herausforderungen, organisationalen und individuellen Ressourcen sowie des Zusammenhangs mit der Arbeitsfähigkeit können als Grundlage dienen für eine menschengerechte Gestaltung von Arbeitssystemen je nach den spezifischen Bedingungen in den jeweiligen Berufsgruppen (s. Tab. 4). Gemäß des Ansatzes soziotechnischer Systeme und der daraus hervorgehenden Mensch-Technik-Organisations- (MTO-) Analyse stehen technische und soziale Aspekte in einem wechselseitigen Bedingungsverhältnis (Rothe et al. 2017). Empfehlungen zur Gestaltung der Arbeitssysteme sollten dies berücksichtigen. Auch die vorliegenden Analysen heben die Bedeutung dieser Bedingungskonstellation hervor. So lassen sich für beide untersuchten Berufsgruppen nicht nur bei der technischen Gestaltung der Arbeitssysteme, sondern vor allem mit Blick auf organisationale Ressourcen Ansatzpunkte für Interventionen ausmachen. Die Arbeitsfähigkeit der Beschäftigten ließe sich bereits mit Hilfe scheinbar ",weicher" Maßnahmen verbessern: die Stärkung sozialer Unterstützung durch Kollegen und Führungskräfte sowie ein auf Anerkennung und Gleichheit als auch auf Rollenklarheit 
basierendes Betriebsklima. Führungskräfte spielen hierbei als Moderatoren und Gestalter eine besondere Rolle. Aufgrund der möglichen Divergenz der Perspektiven sollten Gestaltungsmaßnahmen unter Beteiligung der Beschäftigten eingerichtet werden. Aber auch die Arbeitsplatzanforderungen als solche, vor allem in Form eines starken Termin- und Leistungsdrucks, sind äußerst bedeutend für die Arbeitsfähigkeit. Maßnahmen können sich hier darauf richten, den Arbeitsfluss zu verbessern, ohne die Intensität zu erhöhen. Dies gilt ebenfalls hinsichtlich störender Unterbrechungen in den Postberufen. Schließlich sollte die Infrastruktur der Informations- und Kommunikationstechnologie in der Lagerwirtschaft so gestaltet werden, dass die Handlungsautonomie gestärkt wird.

Der vorliegende Aufsatz soll einen Beitrag zur menschengerechten Gestaltung in den Berufen der Lagerwirtschaft sowie der Post- und Zustelldienste leisten. Allerdings bestehen Einschränkungen, was die Interpretierbarkeit der Ergebnisse betrifft. So sind Kausalaussagen über UrsacheWirkungs-Zusammenhänge aufgrund der Querschnittanalyse eine Frage der Interpretation und nur unter Vorbehalt zu treffen. Es besteht auch insofern weiterhin Forschungsbe-

darf, als dass nur ein Teil der Unterschiede in der Arbeitsfähigkeit in den untersuchten Berufsfeldern erklärt werden konnte. Schließlich wäre zu untersuchen, in welchem Interaktionszusammenhang Anforderungen und Ressourcen stehen. Es ist vor allem die Datenlage, die solche Analysen auf Berufs- oder Betriebsebene schwierig macht.

Förderung Das Forschungsprojekt „Präventive Prinzipien und Methoden der alterns- und marktgerechten Arbeitssystemgestaltung in der Intralogistik" (PREVILOG) wird gefördert durch das Bundesministerium für Bildung und Forschung (BMBF) im Rahmen des Forschungsprogramms „Innovationen für die Produktion Dienstleistung und Arbeit von morgen“ (Fördernummer 02L14A200) und betreut durch den Projektträger Karlsruhe (PTKA).

Funding Open Access funding enabled and organized by Projekt DEAL.

\section{Anhang}

Tab. 5 Vergleich der jeweiligen Berufsgruppen für die Erhebungsjahre 2015 und 2017

Table 5 Comparison of the respective occupational groups for the survey years 2015 and 2017

\begin{tabular}{|c|c|c|c|c|c|c|c|}
\hline \multirow[t]{3}{*}{ Konstrukt } & \multirow[t]{3}{*}{ Item } & \multicolumn{3}{|c|}{ Lagerwirtschaft } & \multicolumn{3}{|c|}{ Post- und Zustelldienst } \\
\hline & & MW & MW & $|r|$ & MW & MW & $|\mathrm{r}|$ \\
\hline & & 2015 & 2017 & & 2015 & 2017 & \\
\hline Arbeitsfähigkeit & Bewertung eigener Arbeitsfähigkeit & 7,21 & 7,26 & 0,01 & 7,49 & 7,52 & 0,03 \\
\hline \multicolumn{8}{|c|}{ Anforderungen: Stressoren } \\
\hline \multirow{3}{*}{$\begin{array}{l}\text { Physische } \\
\text { Tätigkeiten }\end{array}$} & Stehen & 3,70 & 3,75 & 0,05 & 3,68 & 3,67 & 0,01 \\
\hline & Lasten & 2,90 & 2,94 & 0,01 & 3,36 & 3,31 & 0,04 \\
\hline & Kniend & 2,64 & 2,80 & 0,06 & 3,65 & 3,73 & 0,01 \\
\hline \multirow{3}{*}{$\begin{array}{l}\text { Physisches } \\
\text { Arbeitsumfeld }\end{array}$} & Hitze & 2,36 & 2,34 & 0,01 & 2,39 & 2,08 & $0,13^{+}$ \\
\hline & Licht & 1,94 & 1,97 & 0,02 & 2,52 & 2,15 & $0,14^{+}$ \\
\hline & Lärm & 2,59 & 2,77 & 0,06 & 2,33 & 2,17 & 0,06 \\
\hline $\begin{array}{l}\text { Soziales Arbeits- } \\
\text { umfeld }\end{array}$ & Unterbrechung durch Kollegen & 3,04 & 3,22 & $0,08^{+}$ & 2,62 & 2,52 & 0,06 \\
\hline \multirow[t]{2}{*}{ Arbeitszeitlage } & Wochenendarbeit ${ }^{\mathrm{a}}$ & 0,66 & 0,69 & $0,03^{\mathrm{a}}$ & 0,87 & 0,88 & $0,02^{\mathrm{a}}$ \\
\hline & Arbeitszeit außerhalb 7 und $19 \mathrm{Uhr}^{\mathrm{a}}$ & 0,36 & 0,43 & $0,07^{\mathrm{a}}$ & 0,28 & 0,31 & $0,03^{\mathrm{a}}$ \\
\hline \multirow{2}{*}{$\begin{array}{l}\text { Betriebliche } \\
\text { Veränderungen }\end{array}$} & Umstrukturierung $^{\mathrm{a}}$ & 0,41 & 0,38 & $0,03^{\mathrm{a}}$ & 0,55 & 0,58 & $0,03^{\mathrm{a}}$ \\
\hline & Stellenabbau ${ }^{\mathrm{a}}$ & 0,33 & 0,30 & $0,02^{\mathrm{a}}$ & 0,39 & 0,27 & $0,11^{\mathrm{a}}$ \\
\hline \multicolumn{8}{|c|}{ Anforderungen: Herausforderungen } \\
\hline \multirow[t]{3}{*}{ Herausforderungen } & Starker Termin- oder Leistungsdruck & 3,12 & 3,08 & 0,02 & 3,31 & 3,42 & 0,08 \\
\hline & Sehr schnelles Arbeiten & 3,32 & 3,39 & 0,03 & 3,56 & 3,58 & 0,02 \\
\hline & Gleichzeitige Erledigung von Arbeitsabläufen & 3,09 & 3,26 & $0,08^{+}$ & 3,14 & 3,04 & 0,02 \\
\hline \multicolumn{8}{|c|}{ Ressourcen: Organisational } \\
\hline \multirow{6}{*}{$\begin{array}{l}\text { Berufliche } \\
\text { Autonomie }\end{array}$} & Einfluss auf Arbeitsbeginn und -ende & 2,00 & 2,09 & 0,03 & 2,11 & 2,38 & 0,06 \\
\hline & Arbeitsdurchführung vorgeschrieben & 2,97 & 2,92 & 0,02 & 3,11 & 3,02 & 0,04 \\
\hline & Eigene Arbeit selbst planen und einteilen & 3,00 & 3,15 & 0,05 & 3,08 & 3,00 & 0,05 \\
\hline & Einfluss auf Arbeitsmenge & 2,31 & 2,31 & 0,01 & 1,78 & 1,71 & 0,02 \\
\hline & Eigene Entscheidung über Zeitpunkt der Pausen & 2,45 & 2,55 & 0,04 & 3,23 & 3,37 & 0,02 \\
\hline & Einfluss auf Aufgabenart & 2,31 & 2,35 & 0,02 & 1,87 & 1,85 & 0,01 \\
\hline
\end{tabular}


Tab. 5 (Fortsetzung)

Table 5 (Continued)

\begin{tabular}{|c|c|c|c|c|c|c|c|}
\hline \multirow[t]{3}{*}{$\overline{\text { Konstrukt }}$} & \multirow[t]{3}{*}{ Item } & \multicolumn{3}{|c|}{ Lagerwirtschaft } & \multicolumn{3}{|c|}{ Post- und Zustelldienst } \\
\hline & & MW & MW & $|\mathrm{r}|$ & MW & MW & $|\mathrm{r}|$ \\
\hline & & 2015 & 2017 & & 2015 & 2017 & \\
\hline \multirow{3}{*}{$\begin{array}{l}\text { Soziale } \\
\text { Unterstützung: } \\
\text { Kollegen }\end{array}$} & $\begin{array}{l}\text { Gefühl als Teil der Gemeinschaft am Arbeits- } \\
\text { platz }\end{array}$ & 3,27 & 3,42 & 0,06 & 3,25 & 3,20 & 0,03 \\
\hline & Gute Zusammenarbeit mit Kollegen & 3,71 & 3,62 & 0,06 & 3,63 & 3,44 & 0,12 \\
\hline & Unterstützung von Kollegen & 3,55 & 3,59 & 0,02 & 3,39 & 3,27 & 0,06 \\
\hline \multirow{3}{*}{$\begin{array}{l}\text { Soziale } \\
\text { Unterstützung: } \\
\text { Vorgesetzte }\end{array}$} & Unterstützung von Führungskraft (FK) & 2,95 & 2,91 & 0,02 & 2,88 & 2,65 & 0,10 \\
\hline & FK gibt Möglichkeit zur Beteiligung & 2,84 & 2,79 & 0,02 & 2,51 & 2,45 & 0,02 \\
\hline & Rücksichtslose Behandlung durch FK & 1,72 & 1,66 & 0,02 & 1,64 & 1,61 & 0,03 \\
\hline \multicolumn{8}{|c|}{ Ressourcen: Individuell } \\
\hline Bildung & Bildungsniveau & 2,02 & 2,10 & $0,07^{+}$ & 2,04 & 2,04 & 0,00 \\
\hline \multirow[t]{5}{*}{ Selbstwirksamkeit } & $\begin{array}{l}\text { Guter Umgang mit unerwarteten Arbeitssitua- } \\
\text { tionen }\end{array}$ & 3,68 & 4,05 & $0,11 * *$ & 3,73 & 4,17 & $0,15^{*}$ \\
\hline & Gelassenheit bei beruflichen Schwierigkeiten & 3,87 & 4,02 & 0,03 & 4,00 & 4,31 & 0,11 \\
\hline & Ideen zur Problemlösung bei der Arbeit & 3,77 & 4,02 & $0,09 *$ & 3,83 & 4,23 & $0,15^{*}$ \\
\hline & Den Anforderungen im Beruf gewachsen & 4,10 & 4,28 & $0,08^{+}$ & 4,16 & 4,44 & 0,09 \\
\hline & Gut auf berufliche Zukunft vorbereitet & 3,74 & 4,11 & $0,11 * *$ & 3,96 & 4,29 & $0,15^{*}$ \\
\hline \multicolumn{8}{|c|}{ Soziodemografie/Kontrollvariablen } \\
\hline Alter & Alter & 45,05 & 48,40 & $0,14 * * *$ & 48,01 & 49,12 & 0,11 \\
\hline \multirow[t]{2}{*}{ Soziodemografie } & Weibliches Geschlecht ${ }^{\mathrm{a}}$ & 0,36 & 0,36 & $0,00^{\mathrm{a}}$ & 0,52 & 0,44 & $0,07^{\mathrm{a}}$ \\
\hline & Führungskraft ${ }^{\mathrm{a}}$ & 0,23 & 0,20 & $0,04^{\mathrm{a}}$ & 0,17 & 0,13 & $0,04^{\mathrm{a}}$ \\
\hline
\end{tabular}

Hinweis: Mann-Whitney-U-Tests zwischen den Jahren 2015 und 2017 für die Subgruppen Lagerwirtschaft $\left(n_{2015}=348 ; n_{2017}=129\right)$ sowie Postund Zustelldienste $\left(n_{2015}=121 ; n_{2017}=52\right)$

Berechnung von $\mathrm{r}$ folgt der Formel $\mathrm{Z} / \sqrt{(\mathrm{N} x}+\mathrm{Ny})$ (Pallant 2007). $* * * \leq 0,001 ; * * \leq 0,01 ; * \leq 0,05 ;+\leq 0,1$

$M W$ Mittelwert, $r$ Effektstärke

${ }^{\mathrm{a}}=\mathrm{Chi}^{2}$-Test für dichotome Variablen mit Cramers-V als Effektstärke. Nutzung moderner IKT und Betriebsklima 2017 nicht abgefragt

Open Access Dieser Artikel wird unter der Creative Commons Namensnennung 4.0 International Lizenz veröffentlicht, welche die Nutzung, Vervielfältigung, Bearbeitung, Verbreitung und Wiedergabe in jeglichem Medium und Format erlaubt, sofern Sie den/die ursprünglichen Autor(en) und die Quelle ordnungsgemäß nennen, einen Link zur Creative Commons Lizenz beifügen und angeben, ob Änderungen vorgenommen wurden.

Die in diesem Artikel enthaltenen Bilder und sonstiges Drittmaterial unterliegen ebenfalls der genannten Creative Commons Lizenz, sofern sich aus der Abbildungslegende nichts anderes ergibt. Sofern das betreffende Material nicht unter der genannten Creative Commons Lizenz steht und die betreffende Handlung nicht nach gesetzlichen Vorschriften erlaubt ist, ist für die oben aufgeführten Weiterverwendungen des Materials die Einwilligung des jeweiligen Rechteinhabers einzuholen.

Weitere Details zur Lizenz entnehmen Sie bitte der Lizenzinformation auf http://creativecommons.org/licenses/by/4.0/deed.de.

\section{Literatur}

Antonovsky A (1988) Unraveling the mystery of health. How people manage stress and stay well. Jossey-Bass, San Francisco

Baethge A, Vahle-Hinz T, Schulte-Braucks J, van Dick R (2018) A matter of time? Challenging and hindering effects of time pressure on work engagement. Work Stress 32(3):228-247

Bakker AB, Demerouti E (2017) Job demands-resources theory: taking stock and looking forward. J Occup Health Psychol 22(3):273-285
Bundesagentur für Arbeit (2011) Systematischer und alphabetischer Teil mit Erläuterungen. Klassifikation der Berufe 2010, Bd. 1. BA, Nürnberg

Bundesverband Paket und Expresslogistik e. V. (2019) KEP-Studie 2019 - Analyse des Marktes in Deutschland. Berlin: Bundesverband Paket und Expresslogistik e.V. https://www.biek.de/files/ biek/downloads/papiere/BIEK_KEP-Studie_2019.pdf. Zugegriffen: 9. Okt. 2020

Cohen J (1988) Statistical power analysis for the behavioral sciences, 2. Aufl. Lawrence Erlbaum, Hillsdale

Crawford ER, Lepine JA, Rich BL (2010) Linking job demands and resources to employee engagement and burnout: A theoretical extension and meta-analytic test. J Appl Psychol 95(5):834-848

Demerouti E, Bakker AB, Nachreiner F, Schaufeli WB (2001) The job demands-resources model of burnout. J Appl Psychol 86:499-512

Ebener M, Hasselhorn HM (2016) Arbeitsfähigkeit in Organisationen messen und erhalten - ein Konzept und ein Instrument aus der Arbeitsmedizin. Wirtschaftspsychologie 3:48-58

Gimpel H, Lanzl J, Regal C, Urbach N, Wischniewski S, Tegtmeier P, Kreilos M, Kühlmann TM, Becker J, Eimecke J, Derra ND (2019) Gesund digital arbeiten?! Eine Studie zu digitalem Stress in Deutschland. PräDiTec, Augsburg, Dortmund, Bayreuth

Häring A, Schütz H, Gilberg R, Kleudgen M, Wöhrmann AM, Brenscheidt F (2016) Methodenbericht und Fragebogen zur BAuAArbeitszeitbefragung 2015 (baua: Bericht). Dortmund/Berlin/ Dresden: Bundesanstalt für Arbeitsschutz und Arbeitsmedizin (BAuA). https://www.baua.de/DE/Angebote/Publikationen/ Berichte/F2360-2.html. Zugegriffen: 9. Okt. 2020

Häring A, Schütz H, Middendorf L, Hausen J, Brauner C, Wöhrmann AM (2018) Methodenbericht und Fragebogen zur BAuA-Arbeits- 
zeitbefragung 2017 (baua: Bericht). Dortmund/Berlin/Dresden: BAuA. https://www.baua.de/DE/Angebote/Publikationen/Berich te/F2398-3.html. Zugegriffen: 9. Okt. 2020

Härtwig C, Sapronova A (2020) Keine Angst vor der Digitalisierung! Zum Stand digitalisierter Arbeitsanforderungen in verschiedenen Industriebranchen und Tätigkeitsfeldern sowie Zusammenhänge zwischen Belastung, Ressourcen und Beanspruchungsfolgen in Deutschland. Z Arb Wiss. https://doi.org/10.1007/s41449-02000205-y

Hartwig M, Mamrot E (2018) Psychological health and wellbeing in intralogistics workplaces - an empirical analysis. In: Arezes PM, Baptista JS, Barroso MP, Carneiro P, Cordeiro P, Costa N (Hrsg) Occupational Safety and Hygiene VI: Proceedings of the 6th International Symposium on Occupation Safety and Hygiene (SHO 2018) Guimarães, 26-27.03. CRC Press, Boca Raton, S 97-101

Ilmarinen J, Gould R, Järvikoski A, Järvisalo J (2008) Diversity of Work Ability. In: Gould R, Ilmarinen J, Järvisalo J, Koskinen S (Hrsg) Dimensions of work ability: Results of the Health 2000 survey. Finnish Institute of Occupational Health, Helsinki, S 13-24

Knieps F, Pfaff H (2017) Digitale Arbeit - digitale Gesundheit. Zahlen, Daten, Fakten. BKK Gesundheitsreport 2017. Medizinisch Wissenschaftliche Verlagsgesellschaft, Berlin

Korunovska J, Spiekermann S (2019) The effects of digitalization on human energy and fatigue: a review. Vienna University of Economics and Business, Vienna

Lepine JA, Podsakoff NP, Lepine MA (2005) A Meta-analytic test of the challenge stressor-hindrance stressor framework: an explanation for inconsistent relationships among stressors and performance. AMJ 48(5):764-775

Lesener T, Gusy B, Wolter C (2019) The job demands-resources model: a meta-analytic review of longitudinal studies. Work Stress 33(1):76-103

McGonagle AK, Barnes-Farrell JL, Milia L, Fischer F, Hobbs B, IskraGolec I, Kaliterna L, Smith L (2014) Demands, resources, and work ability: a cross-national examination of health care workers. Eur J Work Organ Psychol 23(6):830-846

Melzer M, Hubrich A (2014) Einfluss arbeitsbezogener und individueller Ressourcen auf positive Aspekte der mentalen Gesundheit. BAuA, Dortmund, Berlin, Dresden

Meyer S-C, Hünefeld L (2018) Challenging cognitive demands at work, related working conditions, and employee well-being. IJERPH 15(12):2911

Ortmann U, Walker E (2019) Arbeitsfolgen der Digitalisierung in der Handelslogistik jenseits von Substitution und Aufwertung. Forschungsbericht zum Projekt „Digitale Arbeitsbedingungen in der
Intralogistik des Handels (DiALog)“. Düsseldorf: Forschungsinstitut für gesellschaftliche Weiterentwicklung (e.V.). https:// www.fgw-nrw.de/forschungsergebnisse/forschungsergebnisse/ projektdetails/news/arbeitsfolgen-der-digitalisierung-in-der-han delslogistik-jenseits-von-substitution-und-aufwertung.html. Zugegriffen: 9. Okt. 2020

Pallant J (2007) SPSS survival manual-A step by step guide to data analysis using SPSS for windows. Open University Press, Maidenhead

Rothe I, Adolph L, Beermann B, Schütte M, Windel A, Grewer A, Lenhardt U, Michel J, Thomson B, Formazin M (2017) Psychische Gesundheit in der Arbeitswelt. Wissenschaftliche Standortbestimmung. BAuA, Dortmund, Berlin, Dresden

Schaufeli WB, Taris T (2014) A critical review of the job demandsresources model: implications for improving work and health. In: Bauer GF, Hämmig O (Hrsg) Bridging occupational, organizational and public health: a transdisciplinary approach. Springer, Dordrecht, S 43-68

Schlüter J, Hellmann M, Weyer J (2018) Work Ability und digitale Transformation: zum Spannungsverhältnis steigender Belastungen und abnehmender Ressourcen am Beispiel von Mitarbeitern in der Transportlogistik. AIS Stud 11(2):160-175

Semeijn J, De Waard B, Lambrechts W, Semeijn J (2019) Burning rubber or burning out? The influence of role stressors on burnout among truck drivers. Logistics 3(1):6

Straub N, Kaczmarek S, Hegmanns T, Niehues S (2017) Logistik 4.0 Logistikprozesse im Wandel. Technologischer Wandel in Logistiksystemen und deren Einfluss auf die Arbeitswelt in der operativen Logistik. Ind 40 Manag 33(2):47-51

Thomson B, Steidelmüller C, Schröder T, Wittmers A, Pundt F, Weber C (2020) Der Zusammenhang organisationaler Rahmenbedingungen und Gesundheit bei Führungskräften und Beschäftigten. Projekt F2436 „Führung und Organisation im Wandel“. ASU Z Med Präv 55(Sonderheft): 1-44

Widmer PS, Semmer NK, Kälin W, Jacobshagen N, Meier LL (2012) The ambivalence of challenge stressors: time pressure associated with both negative and positive well-being. J Vocat Behav 80(2):422-433

Van Den Broeck A, De Cuyper N, De Witte H, Vansteenkiste M (2010) Not all job demands are equal: differentiating job hindrances and job challenges in the job demands-resources model. Eur J Work Organ Psychol 19(6):735-759

Zanker C (2018) Branchenanalyse Logistik. Der Logistiksektor zwischen Globalisierung, Industrie 4.0 und Online-Handel. HansBöckler-Stiftung, Düsseldorf 